\title{
A Survey on How Dynamically Changes Topology in Wireless Sensor Network
}

\section{Tamim Al Mahmud}

Computer Science and Engineering, Patuakhali Science and Technology University, BANGLADESH

\begin{abstract}
Wireless sensor networks (WSNs) capable new applications and requires nonconventional paradigms for protocol design due to several constraints. Owing to the prerequisite for low device complexity together with low energy consumption (i.e., long network lifetime), a proper balance between communication and signal/data processing capabilities must be established. This motivates a mammoth endeavour in research performance, standardization procedure, and engineering investments on this field since the last decade. This paper aims at reporting an overview of WSNs technologies, key applications and principles, characteristics in WSNs plan, and evolutions. In scrupulous, several unusual applications, such as those based on ecological monitoring, are discussed and design strategies painted; a case study based on an actual function is also reported. Trends and realistic evolutions are traced. In this paper we have to talk about a range of objectives of existing topology and their limitation. Finally we conclude a proposed solution and its advantage and limitation in focus of our view.
\end{abstract}

Keywords: Wireless sensor networks, exposure area, Connectivity, origin (root) node selection, Energy utilization and Topology of the network nodes.

\section{INTRODUCTION}

A wireless sensor network (WSN) is a collection of distributed autonomous sensors nodes that monitor conditions of physical or environmental equipment, such as vibration, pressure, temperature, sound, pollutants and to jointly transmit their data using the network to a server location. Most of the modern networks are bi-directional, also able to control activity of sensor. The development of WSN was motivated by military applications such that battlefield surveillance; today such types networks are used in many consumer \& industrial application, these are industrial process observing and control, machine health observing, and so on.

The WSN is creating of "nodes" - from several hundreds or even thousands of nodes, where one sensor nodes is connected to each node. Each sensor network consist several parts such as a radio transceiver that has a connection with internal or external aerial, a microcontroller, an electronic path that interfaces with the sensors nodes and an resource of power, usually a battery or an implanted form of energy harvesting. The size of each sensor nodes varies from a shoebox down to the size of a grain of dust. The cost of sensor nodes is generally variable, ranging from several hundreds of dollars, depending on the individual sensor nodes 
complexity. Cost\& size constraints on sensor nodes depend on resources such as energy, memory and communications bandwidth document is a template.

\section{Why Dynamic Topology Changes for Wireless Sensor Network}

In wireless and ad hoc sensor network Topology control is consumed for research community. The principle aim of topology control in the network domain is to save energy, reduce interference between nodes and enhanced lifetime of the network. Increasing lifetime of a sensor node also increase the performance of node to collect and sending information. Wireless sensor network support various topology based on its connectivity to the server. In this paper we basically form a tree topology that is generated on basis of the sink node connected to the ordinary sensor node.

\section{ALGORITHMS FOR TOPOLOGY CONSTRUCTION}

There are many ways to construct topology, they are as follow:

\section{Change the Range of Transmission of the Nodes}

This algorithm represents the clustering of nodes by transmission range based on clustering. In this algorithm topology management is controlled by the usage of coverage area of each node and the power is managed based on mean transmission power within the context of WSN. By reducing the transmission range of the nodes, force devoted by each node is decreased and topology is twisted. Several algorithms are formulated that helps in reducing the network system power consumption and prolonging the battery lifetime of mobile sensor nodes. Formation of cluster and selection of minimal/optimal cluster head and then forming the optimal cluster using weighted metrics like battery lifetime, distance, location and mobility is done on the basis of the facts include node density, coverage surface, contention index, targeted and current node degree of the nodes in the clusters.

Turn off Sensor Nodes from the Network

The Geographical Adaptive Fidelity (GAF) protocol is one kind of methodology to construct dynamic topology. Based on sensor nodes position-awareness, GAF sensor nodes are associated with a squared grid of side (the surface of sensor deployment is divided into a number of these grids). The side of the grid is chosen as a function of the nodes transmission radius, so that any node in a selected grid has a link with any other node in neighbouring grids.

In this case, all nodes in a grid are equivalent. This permits an efficient way for the selection of the node in the grid which stays awake. A leader node is dynamically selected among the sensors in each grid on the basis of nodes residual energy: depending on higher energy, a node is more suitably serve as grid leader. The election procedure is performed by the sensor nodes in accordance with three-state protocol. Every node begins a discovery procedure by which it sets a timer to a time which is inversely proportional to the power. Until control finishing the node carries out a discovery message and moves to the dynamic state. When a node that is whichever in the discovery or in the active state, it receives a discovery message from its nearest node with top energy rank it enters the nap/inactive state, where its radio interface is turned off, for worse energy. By this method, a unique leader is selected in each framework. Selected are the only nodes that remain lively to create the broadcast infrastructure (backbone). Each time a node wakes up and starts again the discovery period that might take to its selection as a new node in charge. By this method, a rotation of the selected node is resulted that leads to reasonable energy utilization at the nodes, only keeping the leader in active mode. 


\section{Generate a broadcast Backbone}

A quite dissimilar solution has come into play a essential role, where the network solidity does not affect the capability of providing a linked network of nodes which are energetic. This code of behaviour is based on the selection of a little portion of the sensor nodes to make a joined backbone, which cover a small part of the total area, which is prepared of backbone nodes and gateways nodes. These backbone nodes are selected in such a system that all node are not in the backbone node.

The selection mechanism follows the fundamental rules of network clustering. In this solution DMAC protocol is proposed to construct backbone. When the protocol operation is started, a DMAC node computes its weight, which indicates how good the node is being for a backbone node. The weight could be computed on the basis of node's residual energy. This also indicates that a node's weight varies in time. Then the node acquires knowledge from its neighbours that which have higher weight. Depending on the weights node decides whether it could be a backbone node or not. This process is completed by periodically sending out "hello" packets that carry the node's identity and current weight. The node with the bigger weight than its neighbourhood will declare that it (node) is going to be a backbone node.

\section{Clustering}

The basic protocol design conception is to reduce all transmissions between sensor nodes linked to nodes invention and the backbone production. While keeping aware all the nodes that compose the connected backbone moreover power saving is gained by transfer to sleep all other ordinary nodes. In S-DMAC just the nodes in the backbone will be wakeful while all the normal nodes are napping. A define scheduling apparatus for a backbone node is to transmit its neighbouring ordinary sensor nodes to nap or inactive.

When the backbone node is expire because of energy reduction. A normal node will be reorganized with a recently inserted backbone node when the power of the new backbone node only exceeds the old node's energy of a predefined capacity. This effectively reduces the fixed cost of the backbone maintenance and it keeps the changes limited. In this process backbone node constitutes clustering.

\section{Problem Identifying in Above Solution(From my Point of View)}

- Cover only limited surface area. Because energy level of certain backbone node is not enough to transmit data at long range.

- Limited lifetime creates problem when requires for longer time transmitting of data.

- Should not properly identify which will initiate or starts the signal that discovers the sensor node.

\section{The Topology supervision Framework}

In this approach every node is familiar to the number of neighbour's nodes that why a complete joined tree-like topology can be constructed headed for the sink-node. A performance handle is used to enables the system designers to construct resultant topologies that are able to be minimized according to numerous traffic samples. This elasticity of topology resolution suggests an efficient traffic between the accessible resources and the network topology. The elementary idea is to employ the energy control mechanism to the topology management where every node is allowed to desire different transmission power stages. The proposal picks a subset of nodes that creates a associated backbone of bi-directional acquaintances towards the sink-node. Every sensor node tries to discover its neighbour node using a MAC signal with the greatest broadcast power. A neighbour counter is maintained in a non-decreasing order of space.

The sink node begins the topology setup stage by turning itself to BLUE and by transfer a topology assembly message with highest transmission power. Among erstwhile fields, the topology building message contains a list smallest distant of neighbour nodes. These 
sections are customized and then broadcast by all other nodes only one time. Upon acceptance the topology construction significance, each WHITE node verifies whether they have symmetric link with the dispatcher node. If the nodes are symmetric the recipient node becomes RED, or else the colour remain same.

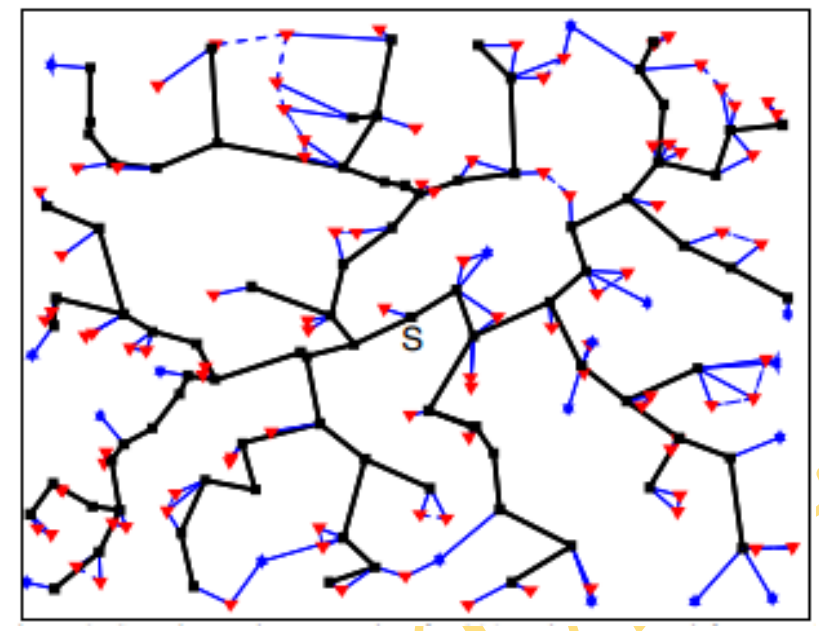

Fig1: Topology management frame work.

\section{Control Dynamic Topology(CDT)}

This advance exchange the advert control messages (ADVERT) throughout the sensor node. The message which dispatch first is the sink node. The transmit advertisement message is transmitted to its specific children. The advertisements are transmitted in the downstream slot of the epoch. During the first epoch a node (not the Sink) is firstly switched on its initialization and sets the whole slot of the node in "scan mode" so that it gain advertisements.

While receiving an advertisement, this packet will send by the MAC to the topology control module running in each node, the packet will process and select randomly to a tree address to be connected. After that the node will transmit a JOIN control packet to the sink-node or the advertiser requesting for a confirmation to the use of specific tree address. When a node receives more than one advertisement it will allow the best RSSI value that is closed to the sink node.

When accepts a join request, the joint acknowledgement (JOIN ACK) will generate by the parent node and deliver it to its child node. After receiving the join acknowledgement the child node changes its status to connect and starts transmitting data upstream and advertising its children (if any). If the node is a terminal node it does not have any child positions so need not to send advertisement. For tracking of the node state each node correspond a list where it keeps information about its children node and free space. During nodes initialization phase, if the node does not receive any advertisement message it keeps all the slots in idle mode and wakes up after listen advertisement messages and repeats the above procedure. 


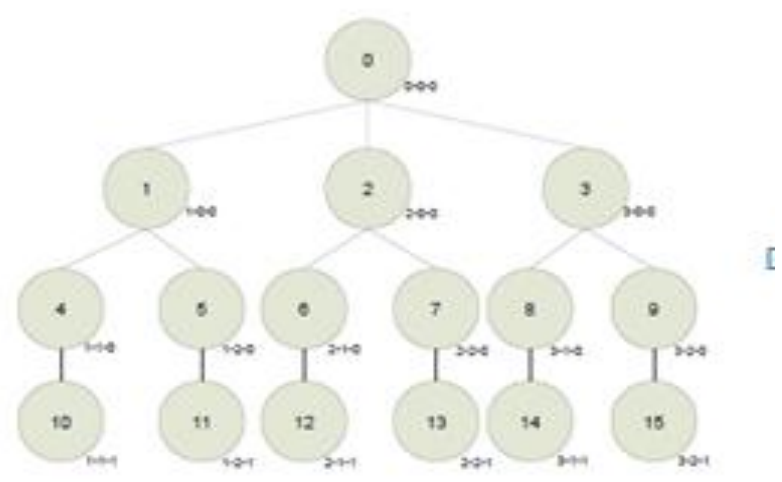

Fig2: Tree topology

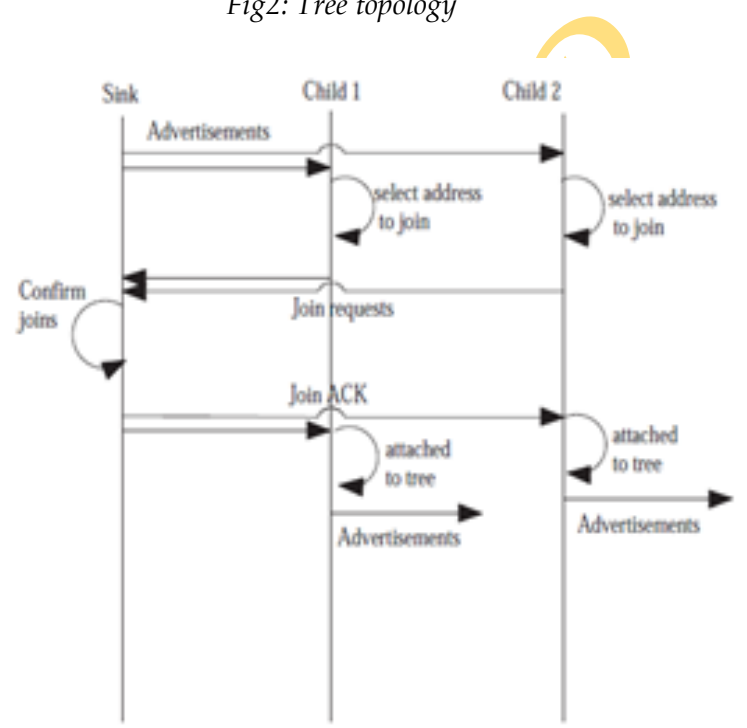

Fig3: Message Sequence Diagram of the Topology Control Mechanism.

\section{Problem Identifying Above Solution}

In the worst case suppose that a certain region would be damaged certain neighbouring node say 10 and 11 has neighbouring node far away from its position. In that case, some active node like 10,11 are with connection less.

\section{A Random Topology with Tree Based Topology Established}

In this approach the blue ellipse is sink-node with surrounding sensor nodes identified by red circle. In this algorithm, the sink node initiates the process and transmits the TEO. It is captured by each node in its range and forwarded it to the next. So the Protocol runs. When a node is reachable through more than one node (dotted link), first the node receives the packet and then replies to its sender. The packet that is received later is simply vanished. So that, each node must know the id of its parent as well as which are of its children. It is easy to compute the total message complexity. For the topology establishment process the complexity is $\mathrm{O}(\mathrm{N})$ where $\mathrm{N}$ is the number of sensor nodes. 


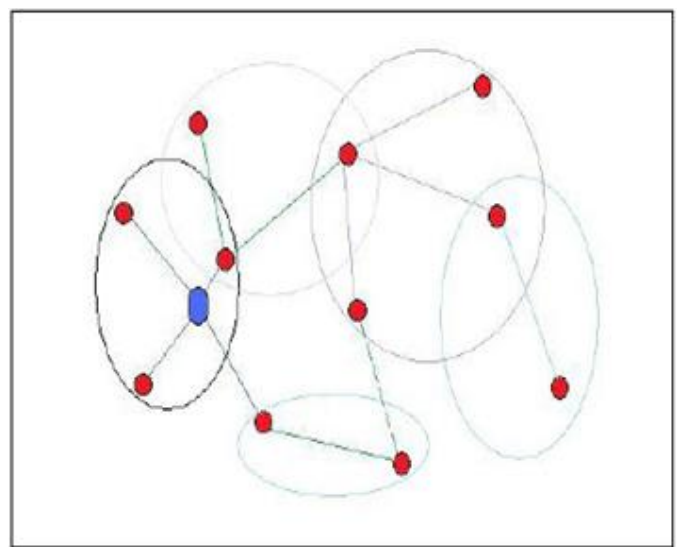

Fig4: Random topology with tree based topology established.

Problem in Random Topology with Tree Based Topology Established (From my Point of View)

- When root node is damaged in certain case then the total transmission system is damaged.

- It is not possible for a node region to transmit data if the distance is long.

- If a certain region is damaged then problem of transmitting data is arises.

- In certain case if two node in the same root node

\section{Proposed Solution}

We proposed that there will be a central node and some supporting node same to the central node whose energy, transmitting power, efficiency all are same to the central node and greater than other sensing nodes. In our proposed topology there are several sink nodes which have greater higher energy then the ordinary sensor node. The sink nodes initiate advertisement randomly. The sensor node receives the advertisement to connect with the sink. The sink node has higher energy that would cover a large territory. The sensor within this territory is connected to the sink node; therefore a large amount of node can be connected. The sensor node in the connected area would be active and sensing it's surrounding and transmit information to the sink. Sink lose its energy after transmitting information.

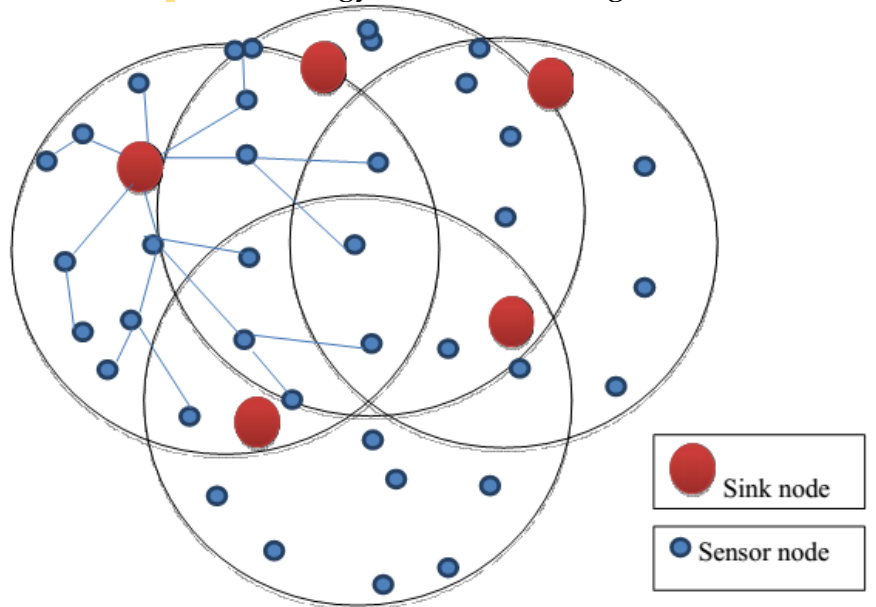

Fig5: Tree topology based on our thinking 
At particular time a particular node will active and collecting sensing node information. After activating for a particular time it may transmit signal to the router and continuously losing its energy. A mechanism is provided after each transmission at central node to check their energy level .Nodes are randomly hand over the mechanism of selecting root node. Repeating this process until all the nodes are damage or transmit information. Then all other nodes are constructing a tree topology with the selecting central node. After selecting central node all other node are construct tree topology with the central node (fig 5). The nodes are transmitting information to the central node and it transmits to the router. Sensing node transmit signal to the root node. The root node covers surface area supporting by its sensing node. Once the root will damage which serve for transmitting the enter network is not disconnected yet. But root node automatically hand over its mechanism to the other root node which will inactive/sleep moment before and collect/gather information coming from sensing node. Sensing node connect/communicate with each other by knowing its neighbor.The discovery of the neighbor nodes (parent and children) is done by the exchange of advertisement control messages (ADVT). The first node that starts sending advertisement messages is the sink node. The advertisements are broadcast messages that advertise specific children tree location. The advertisements are sent in the downstream slot of the epoch. When a node (not the sink) is firstly switched on it initializes during the first epoch and sets all the slots of the node in scan mode so that to receive advertisement.

\section{Conclusions}

Wireless sensor networks become more popular now days because of its low cost, less power requirement, performance and high potential application areas. This paper elaborates the deploy node characteristics and functioning of each module of WSN topology. Although a great work has been done in relation with wireless sensor networks, till date and still many efforts are needed in the direction of design and security of WSN.

\section{REFERENCES}

B. Chen, K. Jamieson, H. Balakrishnan, and R. Morris, "SPAN: An energy efficient coordination algorithm for topology maintenance in ad hoc wireless networks," ACM/Kluwer Wireless Networks, 2002.

Basagni S.,Carosi A.,Petrioli C. Sensor-DMAC: Dynamic Topology Control for Wireless Sensor Networks.

Control with WirelessHART, Hart Communication Foundation Technical ReportHCFLIT-127, March 5, 2010.

Hameed Mir Z.,BaeKo Y. A Topology Management Framework for Wireless Sensor Networks via Power Control.

I.F. Akyildiz, Su Weilian, Y. Sankarasubramaniam, and E. Cayirci, "A Survey on Sensor Networks", IEEE Communications Magazine, August 2002, Vol.: 40, Issue: 8, pp. 102-114.

KoustubhKulkarni, SudipSanyal, Hameed Al-Qaheri, SugataSanyal," Dynamic Reconfiguration of Wireless Sensor Networks" International Journal of Computer Science and Applications Technomathematics Research Foundation Vol. 6, No. 4, pp 16-42, 2009.

Romer, K., Mattern, F.(2004). The Design Space of Wireless Sensor Network.IEEE Wireless Communications, 11(6): 54-61.

Y. Hu, J. Heidemann, and D. Estrin, "Geography-informed energy con-servation for ad hoc routing," in Proceedings of the 7th ACM Annual International Conference on Mobile Computing and Networking, Rome, Italy, July 16-21 2001, pp. 70-84.

ZinonZinonos, VasosVassiliou, Christiana Ioannou, and MariosKoutroullos," Dynamic Topology Control for WSNs in Critical Environments" March 2010, full international standard (IEC 62591Ed. 1.0). 